TRANSACTIONS OF THE

AMERICAN MATHEMATICAL SOCIETY

Volume 364, Number 5, May 2012, Pages 2783-2802

S 0002-9947(2011)05558-3

Article electronically published on December 1, 2011

\title{
POLYGONAL HOMOGRAPHIC ORBITS OF THE CURVED $n$-BODY PROBLEM
}

\author{
FLORIN DIACU
}

\begin{abstract}
In the 2-dimensional $n$-body problem, $n \geq 3$, in spaces of constant curvature, $\kappa \neq 0$, we study polygonal homographic solutions. We first provide necessary and sufficient conditions for the existence of these orbits and then consider the case of regular polygons. We further use this criterion to show that, for any $n \geq 3$, the regular $n$-gon is a polygonal homographic orbit if and only if all masses are equal. Then we prove the existence of relative equilibria of nonequal masses on the sphere of curvature $\kappa>0$ for $n=3$ in the case of scalene triangles. Such triangular relative equilibria occur only along fixed geodesics and are generated from fixed points of the sphere. Finally, through a classification of the isosceles case, we prove that not any choice of three masses can form a triangular relative equilibrium.
\end{abstract}

\section{INTRODUCTION}

We study here the curved $n$-body problem, defined as the motion of $n$ point particles of masses $m_{1}, m_{2}, \ldots, m_{n}>0$ in spaces of constant curvature, $\kappa \neq 0$, under the influence of a natural extension of Newton's gravitational law. The potential defining this law is given by the force function $U_{\kappa}$, whose expression appears in (41). The corresponding potential, given by $-U_{\kappa}$, preserves the basic properties of its Euclidean analogue: it is a harmonic function in 3-dimensional space, i.e., it satisfies Laplace's equation, and generates a central field in which bounded orbits are closed, 24, in agreement with an old result proved by Joseph Louis Bertrand for the Euclidean case, 2].

The research direction we are following here started in the 1830s, when János Bolyai and Nikolai Lobachevsky independently proposed a curved 2-body problem in the hyperbolic space $\mathbf{H}^{3}$, given by a force proportional to the inverse of the area of the sphere of radius equal to the distance between bodies. This problem was studied by top mathematicians, such as Lejeune Dirichlet, Ernest Schering, 31, 32, Wilhelm Killing, [18, [19, 20], and Heinrich Liebmann, 23, [24, 25]. Schering was the one who came up with an analytic form of the potential, which can be expressed in terms of the cotangent of the distance, for $\kappa>0$, and the hyperbolic cotangent of the distance, for $\kappa<0$. The Newtonian law is recovered when $\kappa \rightarrow 0$. Recently, José Cariñena, Manuel Rañada, and Mariano Santander, $[3$, proved several new results for this 2-body problem and revisited some old properties with the help of modern methods. The study of the quantum analogue of the curved 2-body problem was proposed by Erwin Schrödinger, 33, and continued by Leopold Infeld, [16], and Alfred Schild, [17].

Received by the editors October 26, 2010 and, in revised form, January 20, 2011.

2010 Mathematics Subject Classification. Primary 70F10; Secondary 34C25, 37J45.

(C)2011 American Mathematical Society

Reverts to public domain 28 years from publication 
Other attempts at extending the Newtonian case to spaces of constant curvature, such as the efforts of Rudolph Lipschitz in 26], did not survive, mostly because the proposed potentials lacked the basic physical properties mentioned above. Unlike Liebmann, who showed that all the fundamental orbits of the Kepler problem have analogues in curved space, Lipschitz could not obtain explicit solutions; he only succeeded in expressing the orbits in terms of elliptic integrals.

In the direction of research proposed by Bolyai and Lobachevsky, the generalization of the equations of motion to any $n \geq 2$ was given in [7, a paper posted in the arXiv, but submitted for publication as two connected papers, [8] and [9], in which we obtained a unified framework for approaching the problem for any $\kappa \neq 0$. We also proved there the existence of several classes of relative equilibria, including the Lagrangian orbits, i.e. the case $n=3$ of the equilateral triangle. Relative equilibria are orbits for which the configuration of the system remains congruent with itself for all time, so the mutual distances between bodies are constant during the motion.

It is worth mentioning that the study of the curved $n$-body problem, for $n \geq 3$, might help us better understand the nature of the physical space. Gauss allegedly tried to determine the geometry of the universe by measuring the angles of a triangle formed by the peaks of three mountains. Even if the goal of his topographic measurements was different from what anecdotal history attributes to him (see 28), this method of deciding the nature of space remains valid for astronomical distances. But since we cannot measure the angles of cosmic triangles, we could alternatively check whether certain (potentially observable) celestial motions occur in the universe, and thus decide whether the physical space has negative, zero, or positive curvature.

Specifically, we showed in [7] and [8 that while Lagrangian orbits of nonequal masses are known to occur for $\kappa=0$, they must have equal masses for $\kappa \neq 0$. Since Lagrangian solutions of nonequal masses exist in our solar system (such as the triangle formed by the Sun, Jupiter, and the Trojan asteroids), we can conclude that, if assumed to have constant curvature, the physical space is Euclidean for distances of the order $10^{1}$ AU. The discovery of new orbits of the curved $n$-body problem might help us better understand the large-scale geometry of the universe.

The most recent papers on the curved $n$-body problem deal either with singularities, [5], 9], a subject we will not approach here, or with homographic solutions and, in particular, with homothetic orbits and relative equilibria, [7, 9], 6]. Homographic solutions are orbits whose configuration remains similar to itself all along the motion. In particular, when rotation takes place without expansion or contraction, the homographic orbits are called relative equilibria, as mentioned earlier. They behave like rigid bodies, maintaining constant mutual distances. Homothetic solutions are homographic orbits that experience expansion and/or contraction, but no rotation.

The homograpic solutions can be put in a broader perspective. They are also the object of Saari's conjecture, 30, [10, which we partially solved for the curved $n$-body problem, 7], 8], as well as Saari's homographic conjecture, 30, 11. Both have recently generated a lot of interest in classical celestial mechanics (see the references in [10, [11) and are still unsolved in general.

In the classical Newtonian case, 34, as well as in more general classical contexts, 4, the standard concept for understanding homographic solutions is that of central configuration. We will not employ it here since most computations appear 
to be simpler without using it. The reason for these complications is connected to the absence of the integrals of the centre of mass and linear momentum from the curved $n$-body problem. These integrals seem to be specific only to Euclidean space. Indeed, $n$-body problems derived by discretizing Einstein's field equations, as obtained by Tullio Levi-Civita, 21], 22, Albert Einstein, Leopold Infeld, and Banesh Hoffmann, 12, and Vladimir Fock, 14, also lack such integrals.

In this paper we study polygonal homographic orbits of the 2-dimensional curved $n$-body problem. In Section 2, we introduce the notation and the equations of motion as well as their first integrals. In Section 3, we define polygonal homographic orbits and their basic particular cases: homothetic orbits and relative equilibria. We also provide a motivation for our definitions, which are given in the spirit of the Euclidean case. In Section 4, we state and prove necessary and sufficient conditions for the existence of polygonal homographic orbits. Section 5 is dedicated to the study of regular polygons. We show, on the one hand, that if the masses are equal, the regular $n$-gon is a solution of the equations of motion for any $n \geq 3$. On the other hand, we prove that regular $n$-gons can be solutions only when the masses are equal. These results extend the Perko-Walter-Elmabsout theorem, [29], [13], to spaces of nonzero constant curvature. In Section 6 , we focus on the case $n=3$ and prove that the equations of motion admit no homographic orbits if the triangle is not equilateral. For $\kappa>0$ this result is true as long as the homographic orbit is not a relative equilibrium rotating along a great circle of the sphere. In Section 7, we discuss the orbits omitted in the previous section, namely the relative equilibria that move along a geodesic of the sphere. We prove the existence of relative equilibria of nonequal masses in the case of scalene triangles. Such triangular relative equilibria occur only along fixed geodesics and are generated from fixed points on the sphere. Finally we show that not any choice of three masses can form a triangular relative equilibrium by providing a large class of counterexamples in the isosceles case.

\section{Equations of MOTION}

In this section we introduce the equations of motion of the curved $n$-body problem on 2-dimensional manifolds of constant curvature, namely spheres embedded in $\mathbb{R}^{3}$, for $\kappa>0$, and the upper sheets of hyperboloids of two sheet 1 embedded in the Minkowski space $\mathbb{R}^{2,1}$, for $\kappa<0$.

Consider the point particles (bodies) of masses $m_{1}, m_{2}, \ldots, m_{n}>0$ in $\mathbb{R}^{3}$, for $\kappa>0$, and in $\mathbb{R}^{2,1}$, for $\kappa<0$, whose positions are given by the vectors $\mathbf{q}_{i}=$ $\left(x_{i}, y_{i}, z_{i}\right), i=\overline{1, n}$. Let $\mathbf{q}=\left(\mathbf{q}_{1}, \mathbf{q}_{2}, \ldots, \mathbf{q}_{n}\right)$ be the configuration of the system, and $\mathbf{p}=\left(\mathbf{p}_{1}, \mathbf{p}_{2}, \ldots, \mathbf{p}_{n}\right)$, with $\mathbf{p}_{i}=m_{i} \dot{\mathbf{q}}_{i}, i=\overline{1, n}$, representing the momentum. We define the gradient operator with respect to the vector $\mathbf{q}_{i}$ as

$$
\widetilde{\nabla}_{\mathbf{q}_{i}}=\left(\partial_{x_{i}}, \partial_{y_{i}}, \sigma \partial_{z_{i}}\right)
$$

where $\sigma$ is the signum function,

$$
\sigma= \begin{cases}+1, & \text { for } \kappa>0 \\ -1, & \text { for } \kappa<0\end{cases}
$$

\footnotetext{
${ }^{1}$ The upper sheet of the hyperboloid of two sheets corresponds to Weierstrass's model of hyperbolic geometry (see Appendix in 7] or [8]).
} 
and let $\widetilde{\nabla}=\left(\widetilde{\nabla}_{\mathbf{q}_{1}}, \widetilde{\nabla}_{\mathbf{q}_{2}}, \ldots, \widetilde{\nabla}_{\mathbf{q}_{n}}\right)$. For the 3 -dimensional vectors $\mathbf{a}=\left(a_{x}, a_{y}, a_{z}\right)$ and $\mathbf{b}=\left(b_{x}, b_{y}, b_{z}\right)$, we define the inner product

$$
\mathbf{a} \odot \mathbf{b}:=\left(a_{x} b_{x}+a_{y} b_{y}+\sigma a_{z} b_{z}\right)
$$

and the cross product

$$
\mathbf{a} \otimes \mathbf{b}:=\left(a_{y} b_{z}-a_{z} b_{y}, a_{z} b_{x}-a_{x} b_{z}, \sigma\left(a_{x} b_{y}-a_{y} b_{x}\right)\right) .
$$

The Hamiltonian function of the system describing the motion of the $n$-body problem in spaces of constant curvature is

$$
H_{\kappa}(\mathbf{q}, \mathbf{p})=T_{\kappa}(\mathbf{q}, \mathbf{p})-U_{\kappa}(\mathbf{q}),
$$

where

$$
T_{\kappa}(\mathbf{q}, \mathbf{p})=\frac{1}{2} \sum_{i=1}^{n} m_{i}^{-1}\left(\mathbf{p}_{i} \odot \mathbf{p}_{i}\right)\left(\kappa \mathbf{q}_{i} \odot \mathbf{q}_{i}\right)
$$

defines the kinetic energy and

$$
U_{\kappa}(\mathbf{q})=\sum_{1 \leq i<j \leq n} \frac{m_{i} m_{j}|\kappa|^{1 / 2} \kappa \mathbf{q}_{i} \odot \mathbf{q}_{j}}{\left[\sigma\left(\kappa \mathbf{q}_{i} \odot \mathbf{q}_{i}\right)\left(\kappa \mathbf{q}_{j} \odot \mathbf{q}_{j}\right)-\sigma\left(\kappa \mathbf{q}_{i} \odot \mathbf{q}_{j}\right)^{2}\right]^{1 / 2}}
$$

is the force function, $-U_{\kappa}$ representing the potential energy 2 Then the Hamiltonian form of the equations of motion is given by the system

$$
\left\{\begin{array}{l}
\dot{\mathbf{q}}_{i}=m_{i}^{-1} \mathbf{p}_{i}, \\
\dot{\mathbf{p}}_{i}=\widetilde{\nabla}_{\mathbf{q}_{i}} U_{\kappa}(\mathbf{q})-m_{i}^{-1} \kappa\left(\mathbf{p}_{i} \odot \mathbf{p}_{i}\right) \mathbf{q}_{i}, \quad i=\overline{1, n}, \kappa \neq 0,
\end{array}\right.
$$

where the gradient of the force function has the expression

$$
\widetilde{\nabla}_{\mathbf{q}_{i}} U_{\kappa}(\mathbf{q})=\sum_{\substack{j=1 \\ j \neq i}}^{n} \frac{m_{i} m_{j}|\kappa|^{3 / 2}\left(\kappa \mathbf{q}_{j} \odot \mathbf{q}_{j}\right)\left[\left(\kappa \mathbf{q}_{i} \odot \mathbf{q}_{i}\right) \mathbf{q}_{j}-\left(\kappa \mathbf{q}_{i} \odot \mathbf{q}_{j}\right) \mathbf{q}_{i}\right]}{\left[\sigma\left(\kappa \mathbf{q}_{i} \odot \mathbf{q}_{i}\right)\left(\kappa \mathbf{q}_{j} \odot \mathbf{q}_{j}\right)-\sigma\left(\kappa \mathbf{q}_{i} \odot \mathbf{q}_{j}\right)^{2}\right]^{3 / 2}} .
$$

The motion is confined to the surface of nonzero constant curvature $\kappa$, i.e. $(\mathbf{q}, \mathbf{p}) \in$ $\mathbf{T}^{*}\left(\mathbf{M}_{\kappa}^{2}\right)^{n}$, where $\mathbf{T}^{*}\left(\mathbf{M}_{\kappa}^{2}\right)^{n}$ is the cotangent bundle of the configuration space $\left(\mathbf{M}_{\kappa}^{2}\right)^{n}$, and

$$
\mathbf{M}_{\kappa}^{2}=\left\{(x, y, z) \in \mathbb{R}^{3} \mid \kappa\left(x^{2}+y^{2}+\sigma z^{2}\right)=1\right\} .
$$

In particular, $\mathbf{M}_{1}^{2}=\mathbf{S}^{2}$ is the 2-dimensional sphere, and $\mathbf{M}_{-1}^{2}=\mathbf{H}^{2}$ is the 2dimensional hyperbolic plane, represented by the upper sheet of the hyperboloid of two sheets (see the Appendix of [7] or [8] for more details). We will also denote $\mathbf{M}_{\kappa}^{2}$ by $\mathbf{S}_{\kappa}^{2}$ for $\kappa>0$ and by $\mathbf{H}_{\kappa}^{2}$ for $\kappa<0$.

Notice that the $n$ constraints given by $\kappa \mathbf{q}_{i} \odot \mathbf{q}_{i}=1, i=\overline{1, n}$, imply that $\mathbf{q}_{i} \odot \mathbf{p}_{i}=$ 0 , so the $6 n$-dimensional system (5) has $2 n$ constraints. The Hamiltonian function provides the integral of energy,

$$
H_{\kappa}(\mathbf{q}, \mathbf{p})=h,
$$

where $h$ is the energy constant. Equations (5) also have the three integrals of the angular momentum,

$$
\sum_{i=1}^{n} \mathbf{q}_{i} \otimes \mathbf{p}_{i}=\mathbf{c}
$$

\footnotetext{
${ }^{2}$ In [7] and [8], we showed how this expression of $U_{\kappa}$ follows from the cotangent potential for $\kappa \neq 0$, and that $U_{0}$ is the Newtonian potential of the Euclidean problem, obtained as $\kappa \rightarrow 0$.
} 
where $\mathbf{c}=(\alpha, \beta, \gamma)$ is a constant vector. Unlike in the Euclidean case, there are no integrals of the center of mass and linear momentum. Their absence complicates the study of the problem since many of the standard methods don't apply anymore.

Using the fact that $\kappa \mathbf{q}_{i} \odot \mathbf{q}_{i}=1$ for $i=\overline{1, n}$, we can write system (5) as

$$
\ddot{\mathbf{q}}_{i}=\sum_{\substack{j=1 \\ j \neq i}}^{n} \frac{m_{j}|\kappa|^{3 / 2}\left[\mathbf{q}_{j}-\left(\kappa \mathbf{q}_{i} \odot \mathbf{q}_{j}\right) \mathbf{q}_{i}\right]}{\left[\sigma-\sigma\left(\kappa \mathbf{q}_{i} \odot \mathbf{q}_{j}\right)^{2}\right]^{3 / 2}}-\left(\kappa \dot{\mathbf{q}}_{i} \odot \dot{\mathbf{q}}_{i}\right) \mathbf{q}_{i}, \quad i=\overline{1, n},
$$

which is the form of the equations of motion we will use in this paper. The sums on the right hand side of the above equations represent the gradient of the potential. When $\kappa \rightarrow 0$, both the sphere (for $\kappa \rightarrow 0, \kappa>0$ ) and the upper sheet of the hyperboloid of two sheets (for $\kappa \rightarrow 0, \kappa<0$ ) become planes at infinity, relative to the centre of the frame. The segments through the origin of the frame whose angle measures the distance between two points on the curved surface become parallel and infinite, so the distance in the limit plane is the Euclidean distance. Consequently the potential tends to the Newtonian potential as $\kappa \rightarrow 0$ (see [7] or [8] for more details). The terms involving the velocities occur because of the constraints imposed by the curvature. They vanish when $\kappa \rightarrow 0$.

\section{Polygonal homographic orbits}

In this section we define the polygonal homographic solutions of the curved $n$-body problem as well as two remarkable subclasses of solutions: the polygonal homothetic orbits and the polygonal relative equilibria. Then we justify the content of these definitions.

Definition 1. A solution of equations (8), which describe the curved $n$-body problem, is called polygonal homographic if the bodies of masses $m_{1}, m_{2}, \ldots, m_{n}>0$, with $n \geq 3$, form a polygon that is orthogonal to the $z$-axis and remains similar to itself for all time $t$.

According to Definition [1 the size of a polygonal homographic solution can vary, but its shape remains the same. Notice that we imposed the condition that the plane of the polygon is always perpendicular to the $z$-axis. This condition is equivalent to saying that all masses have the same coordinate $z(t)$, which may vary in time. This condition is not imposed for mere simplicity but because polygonal homographic solutions may not exist without it. Though a complete proof of this conjecture is still eluding us, we will explain later in this section why we think this property is true.

We can represent a polygonal homographic solution of the curved $n$-body problem in the form

$$
\begin{array}{r}
\mathbf{q}=\left(\mathbf{q}_{1}, \ldots, \mathbf{q}_{n}\right), \quad \mathbf{q}_{i}=\left(x_{i}, y_{i}, z_{i}\right), \\
x_{i}=r \cos \left(\omega+\alpha_{i}\right), y_{i}=r \sin \left(\omega+\alpha_{i}\right), z_{i}=z, i=\overline{1, n},
\end{array}
$$

where $0 \leq \alpha_{1}<\alpha_{2}<\cdots<\alpha_{n}<2 \pi$ are constants; the function $z=z(t)$ satisfies $z^{2}=\sigma \kappa^{-1}-\sigma r^{2} ; \sigma$ is the signum function defined in (1); $r:=r(t)$ is the size function; $\omega:=\omega(t)$ is the angular function.

Indeed, for every time $t$, we have that $x_{i}^{2}(t)+y_{i}^{2}(t)+\sigma z_{i}^{2}(t)=\kappa^{-1}, i=\overline{1, n}$, which means that the bodies move on the surface $\mathbf{M}_{\kappa}^{2}$, and the angles between any two bodies, seen from the centre of the circle containing the polygon, are invariant 
in time. Therefore representation (9) of the polygonal homographic orbits agrees with Definition 1 .

Definition 2. A polygonal homographic solution of equations (8), which describe the curved $n$-body problem, is called polygonal homothetic if the polygon having at its vertices the bodies of masses $m_{1}, m_{2}, \ldots, m_{n}>0$, with $n \geq 3$, expands or contracts, but does not rotate around the $z$-axis.

In terms of the representation (9), a polygonal homographic solution is polygonal homothetic if $\omega(t)$ is constant, but $r(t)$ is not. Such orbits occur, for instance, when $n$ bodies of equal masses, lying initially at the vertices of a regular polygon inscribed in a nongeodesic circle of the sphere, are released with zero initial velocities, to end up in a total collision.

Definition 3. A polygonal homographic solution of equations (8), which describe the curved $n$-body problem, is called a polygonal relative equilibrium if the polygon having at its vertices the bodies of masses $m_{1}, m_{2}, \ldots, m_{n}>0$, with $n \geq 3$, rotates around the $z$-axis and maintains fixed mutual distances.

In terms of the representation (9), a polygonal relative equilibrium occurs when $r(t)$ is constant, but $\omega(t)$ is not. These orbits have a rich recent history and have been extensively studied in the general context of geometric mechanics (see, e.g., [1], 27]).

We will further loosely use the terms "dynamical polygon" or "dynamical $n$-gon" to describe any of the polygonal homographic, homothetic, or relative equilibrium orbits that will occur in this paper. These terms will also occur when we check potential solutions, prior to knowing whether they satisfy the equations of motion.

Using the concept of relative equilibrium, we can now provide a justification for introducing the orthogonality condition in Definitions 1, 2, and 3. Consider a polygon formed by the bodies of masses $m_{1}, m_{2}, \ldots, m_{n}>0$, which move on the surface $\mathbf{M}_{k}^{2}$ according to equations (8). Unlike in the representation (9), the plane of the polygon can have any angle (not only $\pi / 2$ ) relative to the $z$-axis. We assume this angle to be constant in time. We assign initial velocities such that the dynamical polygon rotates around the $z$-axis, which passes at all times through the same point inside the polygon. The Principal Axis Theorem (see Appendix in [7] or [8]) guarantees the validity of this scenario without any loss of generality.

Let us now seek necessary conditions for the existence of the above-described motion. Notice that the projection of the polygon to the $(x, y)$-plane is, at any time $t$, a polygon congruent with the projection obtained at $t=0$. We can then describe the motion of the real polygon in terms of the angles of the projected polygon. So let us assume that the relative equilibrium is represented by the coordinates $\mathbf{q}_{i}(t)=\left(x_{i}(t), y_{i}(t), z_{i}(t)\right), i=\overline{1, n}$, given by

$$
x_{i}(t)=r_{i} \cos \left(\Omega t+\alpha_{i}\right), y_{i}(t)=r_{i} \sin \left(\Omega t+\alpha_{i}\right), z_{i}(t)=z_{i} \text { (constant), }
$$

where $0 \leq \alpha_{1}<\alpha_{2}<\cdots<\alpha_{n}<2 \pi, \Omega \neq 0$, and $r_{i}>0$ are constants, $z_{i}^{2}=$ $\sigma \kappa^{-1}-\sigma r_{i}^{2}$, and $\sigma$ is the signum function defined in (11).

For such a solution to exist, it is necessary that the total angular momentum is the zero vector or a vector parallel with the $z$-axis. Otherwise the angularmomentum vector would rotate around the $z$-axis, in violation of the angularmomentum integrals (7). This means that at least the first two components of the 
vector $\sum_{i=1}^{n} m_{i} \mathbf{q}_{i} \otimes \dot{\mathbf{q}}_{i}$ are zero, i.e.

$$
\sum_{i=1}^{n} m_{i}\left(y_{i} \dot{z}_{i}-\dot{y}_{i} z_{i}\right)=\sum_{i=1}^{n} m_{i}\left(x_{i} \dot{z}_{i}-\dot{x}_{i} z_{i}\right)=0 .
$$

Using (10) and the fact that $\Omega \neq 0$, these two equations take the form

$$
\sum_{i=1}^{n} m_{i} r_{i} z_{i} \cos \left(\Omega t+\alpha_{i}\right)=\sum_{i=1}^{n} m_{i} r_{i} z_{i} \sin \left(\Omega t+\alpha_{i}\right)=0 .
$$

In general, the above conditions for the existence of motions described above are not satisfied for all values of $t$. But there are exceptions, such as when all $z_{i}$ (and implicitly all $r_{i}$ ) are equal, all $m_{i}$ are equal, and the polygon is regular. So the case when all $z_{i}$ are equal, which implies orthogonality relative to the $z$-axis, seems like a good point to start from. This position is supported by the proof that Lagrangian solutions must be orthogonal to the z-axis (see [7] or [8]). These remarks justify our choice of the orthogonality condition in the definitions of this section.

\section{NECESSARY AND SUFFICIENT CONDITIONS}

The goal of this section is to state and prove two equivalent criteria that provide necessary and sufficient conditions for the existence of polygonal homographic solutions of the curved $n$-body problem. The first criterion can be expressed as follows.

Criterion 1. Consider $n \geq 3$ bodies of masses $m_{1}, m_{2}, \ldots, m_{n}>0$ moving on the surface $\mathbf{M}_{\kappa}^{2}$. The necessary and sufficient conditions that a solution of the form (9) is a polygonal homographic orbit of equations (8) are given by the equations

$$
\delta_{1}=\delta_{2}=\cdots=\delta_{n} \text { and } \gamma_{1}=\gamma_{2}=\cdots=\gamma_{n},
$$

where

$$
\begin{gathered}
\delta_{i}=\sum_{j=1, j \neq i}^{n} m_{j} \mu_{j i}, \quad \gamma_{i}=\sum_{j=1, j \neq i}^{n} m_{j} \nu_{j i}, \quad i=\overline{1, n}, \\
\mu_{j i}=\frac{1}{c_{j i}^{1 / 2}\left(2-c_{j i} \kappa r^{2}\right)^{3 / 2}}, \quad \nu_{j i}=\frac{s_{j i}}{c_{j i}^{3 / 2}\left(2-c_{j i} \kappa r^{2}\right)^{3 / 2}}, \\
s_{j i}=\sin \left(\alpha_{j}-\alpha_{i}\right), \quad c_{j i}=1-\cos \left(\alpha_{j}-\alpha_{i}\right), \quad i, j=\overline{1, n}, \quad i \neq j,
\end{gathered}
$$

and $0 \leq \alpha_{1}<\alpha_{2}<\ldots<\alpha_{n}<2 \pi$ are constants.

Proof. Let us check in equations (8) a solution of the form (9). For this purpose we first compute that

$$
\begin{gathered}
\dot{x}_{i}=\dot{r} \cos \left(\omega+\alpha_{i}\right)-r \dot{\omega} \sin \left(\omega+\alpha_{i}\right), \quad \dot{y}_{i}=\dot{r} \sin \left(\omega+\alpha_{i}\right)+r \dot{\omega} \cos \left(\omega+\alpha_{i}\right), \\
\dot{z}_{i}=-\sigma r \dot{r}\left(\sigma \kappa^{-1}-\sigma r^{2}\right)^{-1 / 2}, \\
\ddot{x}_{i}=\left(\ddot{r}-r \dot{\omega}^{2}\right) \cos \left(\omega+\alpha_{i}\right)-(r \ddot{\omega}+2 \dot{r} \dot{\omega}) \sin \left(\omega+\alpha_{i}\right), \\
\ddot{y}_{i}=\left(\ddot{r}-r \dot{\omega}^{2}\right) \sin \left(\omega+\alpha_{i}\right)+(r \ddot{\omega}+2 \dot{r} \dot{\omega}) \cos \left(\omega+\alpha_{i}\right), \\
\ddot{z}_{i}=-\sigma r \ddot{r}\left(\sigma \kappa^{-1}-\sigma r^{2}\right)^{-1 / 2}-\kappa^{-1} \dot{r}^{2}\left(\sigma \kappa^{-1}-\sigma r^{2}\right)^{-3 / 2}
\end{gathered}
$$


Some long but straightforward computations for the $\ddot{z}_{i}$ component lead us to the equations

$$
\ddot{r}=r\left(1-\kappa r^{2}\right) \dot{\omega}-\frac{\kappa r \dot{r}^{2}}{1-\kappa r^{2}}-\Delta_{i}, \quad i=\overline{1, n},
$$

where

$$
\Delta_{i}=\sum_{j=1, j \neq i}^{n} \frac{m_{j}\left(1-\kappa r^{2}\right)}{c_{j i}^{1 / 2} r^{2}\left(2-c_{j i} \kappa r^{2}\right)^{3 / 2}}, \quad i=\overline{1, n}
$$

and the constants $c_{j i}$ are defined in (13). Notice that, as long as the bodies stay away from collisions, we have $c_{j i}>0$.

From the equations corresponding to $\ddot{x}_{i}$ and $\ddot{y}_{i}$, we further apply the formula for the cosine of the sum of two angles to $\cos \left(\omega+\alpha_{j}\right)$, viewed as $\cos \left[\left(\omega+\alpha_{i}\right)+\left(\alpha_{j}-\right.\right.$ $\left.\alpha_{i}\right)$ ]. Then, using the fact that the equations must be satisfied for all times $t$, and comparing the similar terms, we recover equations (15) as well as obtain some new equations,

$$
r \ddot{\omega}+2 \dot{r} \dot{\omega}-\Gamma_{i}=0, \quad i=\overline{1, n},
$$

where

$$
\Gamma_{i}=\sum_{j=1, j \neq i}^{n} \frac{m_{j} s_{j i}}{c_{j i}^{3 / 2} r^{2}\left(2-c_{j i} \kappa r^{2}\right)^{3 / 2}}, \quad i=\overline{1, n},
$$

and the constants $s_{j i}, c_{j i}, i, j=\overline{1, n}, i \neq j$, are defined in (13). Notice that the $s_{j i}$ can have any sign, with $s_{j i}=0$ only if $\alpha_{j}-\alpha_{i}= \pm \pi / 2$ or $\pm 3 \pi / 2$.

Equations (15) and (17) describe the motion of the polygonal homographic orbit with the help of the size function, $r$, and the angular function, $\omega$. But this system of differential equations makes sense only if the conditions

$$
\Delta_{1}=\Delta_{2}=\cdots=\Delta_{n} \text { and } \Gamma_{1}=\Gamma_{2}=\cdots=\Gamma_{n}
$$

are satisfied, where the functions $\Delta_{i}, \Gamma_{i}, i=\overline{1, n}$, are defined in (16) and (18), respectively. Then, for any initial conditions $(r(0), \omega(0))$ that are not singular (i.e. are not collisions for any $\kappa \neq 0$, and are not antipodal for $\kappa>0$; see [7] or [9]), we are guaranteed the local existence and uniqueness of an analytic solution for the system given by equations (15) and (17). Since the phase space is a connected manifold, this solution can be analytically extended to a global solution, defined either for all time or until the orbit reaches a singular configuration, as may happen, for instance, in the case of a homothetic orbit that ends in a total collision.

Multiplying equation (15) by $r^{2} /\left(1-\kappa r^{2}\right)$ and equation (17) by $r^{2}$, the conditions for the existence of polygonal homographic orbits reduce to

$$
\delta_{1}=\delta_{2}=\cdots=\delta_{n} \text { and } \gamma_{1}=\gamma_{2}=\cdots=\gamma_{n},
$$

as defined in the above statement. This remark completes the proof.

Remark. Notice that, if $\delta$ denotes any $\delta_{i}$ and $\gamma$ denotes any $\gamma_{i}, i=\overline{1, n}$, the functions $\mu_{j i}$ and $\delta$ are always positive, while $\nu_{j i}$ and $\gamma$ can be negative, positive, or zero.

We can restate Criterion 14 in terms of linear algebraic systems as follows. The equivalence between Criterion 14 and Criterion 2 is obvious. 
Criterion 2. Consider $n \geq 3$ bodies of masses $m_{1}, m_{2}, \ldots, m_{n}>0$ moving on the surface $\mathbf{M}_{\kappa}^{2}$. The necessary and sufficient conditions that a solution of the form (91) is a polygonal homographic orbit of equations (8) are that there exist $\delta>0$ and $\gamma \in \mathbb{R}$ such that the linear systems

$$
\Delta \mathbf{m}^{T}=\mathbf{d}^{T} \text { and } \Gamma \mathbf{m}^{T}=\mathbf{e}^{T}
$$

have the same set of solutions, where

$$
\begin{gathered}
\mathbf{m}=\left(m_{1}, m_{2}, \ldots, m_{n}\right), \quad \mathbf{d}=(\underbrace{\delta, \delta, \ldots, \delta}_{n \text { times }}), \quad \mathbf{e}=(\underbrace{\gamma, \gamma, \ldots, \gamma}_{n \text { times }}) \\
\Delta=\left[\begin{array}{ccccc}
0 & \mu_{21} & \mu_{31} & \ldots & \mu_{n 1} \\
\mu_{12} & 0 & \mu_{32} & \ldots & \mu_{n 2} \\
\mu_{13} & \mu_{23} & 0 & \ldots & \mu_{n 3} \\
\vdots & \vdots & \vdots & & \vdots \\
\mu_{1 n} & \mu_{2 n} & \mu_{3 n} & \ldots & 0
\end{array}\right], \quad \Gamma=\left[\begin{array}{ccccc}
0 & \nu_{21} & \nu_{31} & \ldots & \nu_{n 1} \\
\nu_{12} & 0 & \nu_{32} & \ldots & \nu_{n 2} \\
\nu_{13} & \nu_{23} & 0 & \ldots & \nu_{n 3} \\
\vdots & \vdots & \vdots & & \vdots \\
\nu_{1 n} & \nu_{2 n} & \nu_{3 n} & \ldots & 0
\end{array}\right],
\end{gathered}
$$

$\mu_{j i}, \nu_{j i}, i, j=\overline{1, n}, i \neq j$ are defined in (12), and the upper index ${ }^{T}$ denotes the transpose of a vector or matrix.

Remark. Notice that $\Delta$ is symmetric, i.e. $\Delta^{T}=\Delta$, whereas $\Gamma$ is skew-symmetric, i.e. $\Gamma^{T}=-\Gamma$. In the next section we will see that, for regular polygons, both $\Delta$ and $\Gamma$ are circulant matrices, i.e. each row vector is rotated one element to the right relative to the previous (upper) row vector.

\section{REgular Polygons}

In this section we will study the case of regular polygons and prove that the curved $n$-body problem, with $n \geq 3$, admits regular polygonal homographic orbits if and only if all the masses are equal. This result is valid only for $n \geq 4$ in the Euclidean case, where it is known as the Perko-Walter-Elmabsout theorem. The classical Lagrangian orbits, given by the dynamic equilateral triangle, allow nonequal masses, a situation that seems to be specific to the Euclidean space, most likely because some symmetries are lost if the curvature is not zero (see also 4]). To emphasize certain details of our result, we will separately consider the direct and converse components of this theorem.

Theorem 1. Consider the curved $n$-body problem, $n \geq 3$, given by system (8). If $n$ bodies of equal masses, $m:=m_{1}=m_{2}=\cdots=m_{n}$, lie initially at the vertices of a regular $n$-gon parallel with the $(x, y)$-plane, then there is a class of initial velocities for which the corresponding solutions are homographic. These orbits also satisfy the equalities $\gamma_{1}=\gamma_{2}=\cdots=\gamma_{n}=0$.

Proof. Let the dynamical $n$-gon be regular and, for every time instant, lie in a plane parallel with the $(x, y)$-plane. We will show that $\delta_{1}=\delta_{2}=\cdots=\delta_{n}$ and $\gamma_{1}=\gamma_{2}=\cdots=\gamma_{n}$, identities which, by Criterion [14, assure the existence of the desired homographic solution.

Without loss of generality, we assume that $\alpha_{i}, i=\overline{1, n}$, the constants which provide the representation (9) of the homographic orbits, are

$$
\alpha_{1}=0, \alpha_{2}=2 \pi / n, \alpha_{3}=4 \pi / n, \ldots, \alpha_{n}=2(n-1) \pi / n .
$$


Then the differences $\alpha_{j}-\alpha_{i}, i, j=\overline{1, n}$, with $i \neq j$, can take only the values

$$
\pm 2 \pi / n, \pm 4 \pi / n, \ldots, \pm 2(n-1) \pi / n \text {. }
$$

Consequently, these angles fully determine the constants $s_{i j}$ and $c_{i j}$ defined in (13).

We will first study the matrix $\Delta$. Let $s=[n / 2]$ denote the integer part of $n / 2$. Then, for $n$ odd, the matrix $\Delta$ takes the circulant form

$$
\left[\begin{array}{cccccccccccc}
0 & a_{1} & a_{2} & \ldots & a_{s-1} & a_{s} & a_{s} & a_{s-1} & \ldots & a_{3} & a_{2} & a_{1} \\
a_{1} & 0 & a_{1} & \ldots & a_{s-2} & a_{s-1} & a_{s} & a_{s} & \ldots & a_{4} & a_{3} & a_{2} \\
a_{2} & a_{1} & 0 & \ldots & a_{s-3} & a_{s-2} & a_{s-1} & a_{s} & \ldots & a_{5} & a_{4} & a_{3} \\
\vdots & \vdots & \vdots & & \vdots & \vdots & \vdots & \vdots & & \vdots & \vdots & \vdots \\
a_{s-1} & a_{s-2} & a_{s-3} & \ldots & 0 & a_{1} & a_{2} & a_{3} & \ldots & a_{s-1} & a_{s} & a_{s} \\
a_{s} & a_{s-1} & a_{s-2} & \ldots & a_{1} & 0 & a_{1} & a_{2} & \ldots & a_{s-2} & a_{s-1} & a_{s} \\
a_{s} & a_{s} & a_{s-1} & \ldots & a_{2} & a_{1} & 0 & a_{1} & \ldots & a_{s-3} & a_{s-2} & a_{s-1} \\
a_{s-1} & a_{s} & a_{s} & \ldots & a_{3} & a_{2} & a_{1} & 0 & \ldots & a_{s-4} & a_{s-3} & a_{s-2} \\
\vdots & \vdots & \vdots & & \vdots & \vdots & \vdots & \vdots & & \vdots & \vdots & \vdots \\
a_{3} & a_{4} & a_{5} & \ldots & a_{s-1} & a_{s-2} & a_{s-3} & a_{s-4} & \ldots & 0 & a_{1} & a_{2} \\
a_{2} & a_{3} & a_{4} & \ldots & a_{s} & a_{s-1} & a_{s-2} & a_{s-3} & \ldots & a_{1} & 0 & a_{1} \\
a_{1} & a_{2} & a_{3} & \ldots & a_{s} & a_{s} & a_{s-1} & a_{s-2} & \ldots & a_{2} & a_{1} & 0
\end{array}\right],
$$

whereas for $n$ even, $\Delta$ becomes the circulant matrix

$$
\left[\begin{array}{ccccccccccc}
0 & a_{1} & a_{2} & \ldots & a_{s-1} & a_{s} & a_{s-1} & \ldots & a_{3} & a_{2} & a_{1} \\
a_{1} & 0 & a_{1} & \ldots & a_{s-2} & a_{s-1} & a_{s} & \ldots & a_{4} & a_{3} & a_{2} \\
a_{2} & a_{1} & 0 & \ldots & a_{s-3} & a_{s-2} & a_{s-1} & \ldots & a_{5} & a_{4} & a_{3} \\
\vdots & \vdots & \vdots & & \vdots & \vdots & \vdots & & \vdots & \vdots & \vdots \\
a_{s-1} & a_{s-2} & a_{s-3} & \ldots & 0 & a_{1} & a_{2} & \ldots & a_{s-2} & a_{s-1} & a_{s} \\
a_{s} & a_{s-1} & a_{s-2} & \ldots & a_{1} & 0 & a_{1} & \ldots & a_{s-3} & a_{s-2} & a_{s-1} \\
a_{s-1} & a_{s} & a_{s-1} & \ldots & a_{2} & a_{1} & 0 & \ldots & a_{s-4} & a_{s-3} & a_{s-2} \\
\vdots & \vdots & \vdots & & \vdots & \vdots & \vdots & & \vdots & \vdots & \vdots \\
a_{3} & a_{4} & a_{5} & \ldots & a_{s-2} & a_{s-3} & a_{s-4} & \ldots & 0 & a_{1} & a_{2} \\
a_{2} & a_{3} & a_{4} & \ldots & a_{s-1} & a_{s-2} & a_{s-3} & \ldots & a_{1} & 0 & a_{1} \\
a_{1} & a_{2} & a_{3} & \ldots & a_{s} & a_{s-1} & a_{s-2} & \ldots & a_{2} & a_{1} & 0
\end{array}\right],
$$

where $a_{1}, a_{2}, \ldots, a_{s}>0$ represent the $\mu_{j i}$ functions in their corresponding positions. Notice that in the $i$ th row, the functions $\mu_{j i}$ with $|i-j|=s$ and $|i-j|=n-s$, $s=\overline{1,[n / 2]}$, are equal, and they are independent of $i$. But in every row of $\Delta$, there are exactly two functions $\mu_{j i}$ with $|i-j|=s$ or $|i-j|=n-s, s=\overline{1,[n / 2]}$. For $n$ even, the single $\mu_{j i}$ term not captured in the above description corresponds to $\alpha_{j}-\alpha_{i}=\pi$ and is of the form $a_{s}=1 /\left[4\left(1-\kappa r^{2}\right)^{3 / 2}\right]$, so it is also independent of $i$. Consequently each row of $\Delta$ contains the same elements, only differently ordered. Since all the masses are equal, we can conclude that $\delta_{1}=\delta_{2}=\cdots=\delta_{n}$.

For $n$ odd, the matrix $\Gamma$ is circulant,

$$
\left[\begin{array}{cccccccccccc}
0 & b_{1} & b_{2} & \ldots & b_{s-1} & b_{s} & -b_{s} & -b_{s-1} & \ldots & -b_{3} & -b_{2} & -b_{1} \\
-b_{1} & 0 & b_{1} & \ldots & b_{s-2} & b_{s-1} & b_{s} & -b_{s} & \cdots & -b_{4} & -b_{3} & -b_{2} \\
-b_{2} & -b_{1} & 0 & \ldots & b_{s-3} & b_{s-2} & b_{s-1} & b_{s} & \cdots & -b_{5} & -b_{4} & -b_{3} \\
\vdots & \vdots & \vdots & & \vdots & \vdots & \vdots & \vdots & & \vdots & \vdots & \vdots \\
-b_{s-1} & -b_{s-2} & -b_{s-3} & \ldots & 0 & b_{1} & b_{2} & b_{3} & \ldots & b_{s-1} & b_{s} & -b_{s} \\
-b_{s} & -b_{s-1} & -b_{s-2} & \ldots & -b_{1} & 0 & b_{1} & b_{2} & \cdots & b_{s-2} & b_{s-1} & b_{s} \\
b_{s} & -b_{s} & -b_{s-1} & \ldots & -b_{2} & -b_{1} & 0 & b_{1} & \cdots & b_{s-3} & b_{s-2} & b_{s-1} \\
b_{s-1} & b_{s} & -b_{s} & \ldots & -b_{3} & -b_{2} & -b_{1} & 0 & \cdots & b_{s-4} & b_{s-3} & b_{s-2} \\
\vdots & \vdots & \vdots & & \vdots & \vdots & \vdots & \vdots & & \vdots & \vdots & \vdots \\
b_{3} & b_{4} & b_{5} & \ldots & -b_{s-1} & -b_{s-2} & -b_{s-3} & -b_{s-4} & \ldots & 0 & b_{1} & b_{2} \\
b_{2} & b_{3} & b_{4} & \cdots & -b_{s} & -b_{s-1} & -b_{s-2} & -b_{s-3} & \cdots & -b_{1} & 0 & b_{1} \\
b_{1} & b_{2} & b_{3} & \cdots & b_{s} & -b_{s} & -b_{s-1} & -b_{s-2} & \cdots & -b_{2} & -b_{1} & 0
\end{array}\right],
$$


whereas for $n$ even, $\Gamma$ takes the circulant form

$$
\left[\begin{array}{ccccccccccc}
0 & b_{1} & b_{2} & \ldots & b_{s-1} & 0 & -b_{s-1} & \ldots & -b_{3} & -b_{2} & -b_{1} \\
-b_{1} & 0 & b_{1} & \ldots & b_{s-2} & b_{s-1} & 0 & \cdots & -b_{4} & -b_{3} & -b_{2} \\
-b_{2} & -b_{1} & 0 & \ldots & b_{s-3} & b_{s-2} & b_{s-1} & \cdots & -b_{5} & -b_{4} & -b_{3} \\
\vdots & \vdots & \vdots & & \vdots & \vdots & \vdots & & \vdots & \vdots & \vdots \\
-b_{s-1} & -b_{s-2} & -b_{s-3} & \ldots & 0 & b_{1} & b_{2} & \ldots & b_{s-2} & b_{s-1} & 0 \\
0 & -b_{s-1} & -b_{s-2} & \ldots & -b_{1} & 0 & b_{1} & \ldots & b_{s-3} & b_{s-2} & b_{s-1} \\
b_{s-1} & 0 & -b_{s-1} & \ldots & -b_{2} & -b_{1} & 0 & \ldots & b_{s-4} & b_{s-3} & b_{s-2} \\
\vdots & \vdots & \vdots & & \vdots & \vdots & \vdots & & \vdots & \vdots & \vdots \\
b_{3} & b_{4} & b_{5} & \ldots & -b_{s-2} & -b_{s-3} & -b_{s-4} & \ldots & 0 & b_{1} & b_{2} \\
b_{2} & b_{3} & b_{4} & \ldots & -b_{s-1} & -b_{s-2} & -b_{s-3} & \cdots & -b_{1} & 0 & b_{1} \\
b_{1} & b_{2} & b_{3} & \ldots & 0 & -b_{s-1} & -b_{s-2} & \cdots & -b_{2} & -b_{1} & 0
\end{array}\right],
$$

where $b_{1}, b_{2}, \ldots, b_{s}>0$ represent the corresponding $\nu_{j i}$ functions. Thus, for $\Gamma$, we have to slightly amend the arguments used for $\Delta$ : the two functions $\nu_{j i}$ with $|i-j|=s$ or $|i-j|=n-s$, which are independent of $i$ and occur in every row, are equal only in absolute value; they have opposite signs because $s_{j i}:=\sin \left(\alpha_{j}-\alpha_{i}\right)$ is odd. For $n$ even, the extra term corresponding to $\alpha_{j}-\alpha_{i}=\pi$ is $b_{s}=0$. Therefore $\gamma_{1}=\gamma_{2}=\cdots=\gamma_{n}=0$. By Criterion 14, we can now conclude that the dynamical regular $n$-gon having equal masses at its vertices is a homographic orbit of the curved $n$-body problem. This remark completes the proof.

We can now state and prove the converse of Theorem 1

Theorem 2. If the masses $m_{1}, \ldots, m_{n}>0, n \geq 3$, form a polygonal homographic solution of the curved $n$-body problem given by equations (8), such that the polygon is regular, then $m_{1}=m_{2}=\cdots=m_{n}$.

Proof. Notice first that, from the form of the matrix $\Gamma$ represented above (for both $n$ even and odd), if a dynamical regular $n$-gon parallel with the $(x, y)$-plane is a homographic solution of the curved $n$-body problem, then the masses must satisfy the system $\Gamma \mathbf{m}^{T}=\mathbf{0}^{T}$. By Criterion 2, the masses must also satisfy the system $\Delta \mathbf{m}^{T}=\mathbf{d}^{T}$.

Since, in general, the functions $\mu_{j i}$ and $\nu_{j i}$ vary in time, and the solutions $m_{1}, m_{2}, \ldots, m_{n}$ of the systems $\Delta \mathbf{m}^{T}=\mathbf{d}^{T}$ and $\Gamma \mathbf{m}^{T}=\mathbf{0}^{T}$ depend on $\mu_{j i}$ and $\nu_{j i}$, only solutions for which the masses are constant and positive lead to homographic solutions. Therefore if we fix an arbitrary time $t$ and show that the corresponding systems with constant coefficients have solutions only when $m_{1}=m_{2}=\cdots=m_{n}$, then no other solutions are possible for those systems when $t$ varies. By Theorem 1 , we can then conclude that $m_{1}=m_{2}=\cdots=m_{n}$ is the only case when the systems with variable coefficients have solutions.

So let us assume $t$ fixed and start with the system $\Delta \mathbf{m}^{T}=\mathbf{d}^{T}$. We already know from Theorem 1 that this system has infinitely many solutions, namely $m_{1}=m_{2}=$ $\cdots=m_{n}=\alpha$ for any $\alpha>0$, where $\alpha$ is some function of $\delta>0$. But if we fix a value of $\delta$, then $\alpha$ is also fixed.

We will now show that $\operatorname{det} \Delta \neq 0$. To prove this fact, notice that, except for the diagonal elements, all the elements of $\Delta$ are positive. Therefore $\Delta$ is positive definite. Indeed, a simple computation shows that for any nonzero vector $\mathbf{z}=$ $\left(z_{1}, z_{2}, \ldots, z_{n}\right) \in \mathbb{R}^{n}, \mathbf{z} \Delta \mathbf{z}^{T}>0$. By Sylvester's criterion (see, e.g., [15]), positivedefinite matrices have positive determinants.

According to Cramer's rule, the linear system $\Delta \mathbf{m}^{T}=\mathbf{d}^{T}$ has a unique solution, which must be none else than $m_{1}=m_{2}=\cdots=m_{n}=\alpha$, with $\alpha>0$ fixed. By Theorem 1. this solution also satisfies the system $\Gamma \mathbf{m}^{T}=\mathbf{0}^{T}$. Since the choice of 
$\alpha$ depends on the choice of $\delta$, and $\delta>0$ is arbitrarily fixed, we can draw the same conclusion for any choice of $\delta$. Consequently a dynamical regular $n$-gon can be a homographic solution of the curved $n$-body problem only if all masses are equal. This remark completes the proof.

\section{NONGEOdESIC SCALENE TRIANGLES}

It is natural to ask whether irregular polygons could form homographic orbits. The conditions of Criterion 14 for the existence and uniqueness of dynamical $n$-gons suggest that, in general, this is not the case. Indeed, system (11) has $2 n-2$ linear equations and $n$ unknowns: $m_{1}, m_{2}, \ldots, m_{n}$. Even the case $n=3$ leads to a linear system of 4 equations with 3 unknowns, which is still unlikely to have solutions in general.

We will next prove that homographic orbits of the curved 3-body problem cannot exist for $|z| \neq 0$ if the triangle is not equilateral. In other words, the symmetries of the equilateral triangle (and consequently the equality of the masses) are a necessary condition for the existence of such orbits. The restriction $|z| \neq 0$ is necessary only for $\kappa>0$ (being automatically satisfied for $\kappa<0$ ) because equations (15) do not allow homographic orbits to pass through the equator of the sphere, since the function $1-\kappa r^{2}$, which appears as a denominator, cancels in that case. However, as we will see in the next section, nonequilateral relative equilibria moving along the equator do exist.

We can now state and prove the following result.

Theorem 3. Consider the curved 3-body problem, given by equations (8) with $n=3$ and masses $m_{1}, m_{2}, m_{3}>0$. These equations admit no homographic orbits given by scalene nonequilateral triangles for $\kappa<0$. For $\kappa>0$, they don't admit such solutions either if the bodies stay away from the equator $z=0$.

Proof. Notice first that any homographic orbit of the 3-body problem must correspond to an acute triangle. Otherwise, at every time instant, there is a plane containing the $z$-axis such that all three bodies are on one side of the plane (at most two of them in the plane). Therefore the total angular momentum vector at that time instant cannot be zero or parallel with the $z$-axis. But after rotating by $\pi$ radians, the bodies reach a position on the other side of the plane, and the angular momentum has certainly a different direction, in violation of the angular momentum integrals (7).

For the masses $m_{1}, m_{2}, m_{3}>0$, the conditions in Criterion 2 can be written as

$$
\begin{aligned}
\Delta \mathbf{m}^{T} & =\mathbf{d}^{T} \text { and } \Gamma \mathbf{m}^{T}=\mathbf{e}^{T}, \\
\mathbf{m} & =\left(m_{1}, m_{2}, m_{3}\right), \quad \mathbf{d}=(\delta, \delta, \delta), \quad \mathbf{e}=(\gamma, \gamma, \gamma), \\
\Delta & =\left[\begin{array}{lll}
0 & a & b \\
a & 0 & c \\
b & c & 0
\end{array}\right], \quad \Gamma=\left[\begin{array}{ccc}
0 & u & v \\
-u & 0 & w \\
-v & -w & 0
\end{array}\right], \\
a & :=\mu_{21}=\mu_{12}, b:=\mu_{31}=\mu_{13}, c:=\mu_{32}=\mu_{23}, \\
u & :=\nu_{21}=-\nu_{12}, v:=\nu_{31}=-\nu_{13}, w:=\nu_{32}=-\nu_{23},
\end{aligned}
$$

and $\mu_{j i}, \nu_{j i}, i, j=1,2,3, i \neq j$, as defined in (12). 
Multiplying the first equation of the system $\Gamma \mathbf{m}^{T}=\mathbf{e}^{T}$ by $-w$, the second by $v$, and adding them, we obtain the equation

$$
-u v m_{1}-u w m_{2}=\gamma(v-w) .
$$

Multiplying the third equation of that system by $-u$ and adding the above equation to it, we get the condition $\gamma(v-u-w)=0$. Circular permutations lead us to the system of conditions

$$
\gamma(v-u-w)=\gamma(w-v-u)=\gamma(u-w-v)=0 .
$$

This system is satisfied either if $\gamma=0$ or when

$$
v-u-w=w-v-u=u-w-v=0 .
$$

But the above equations imply that $u=v=w=0$, which is a solution with no dynamical consequences, so necessarily $\gamma=0$. Consequently the linear system $\Gamma \mathbf{m}^{T}=\mathbf{e}^{T}$ reduces to

$$
u m_{2}+v m_{3}=-u m_{1}+w m_{3}=-v m_{1}-w m_{2}=0 .
$$

Since $m_{1}, m_{2}, m_{3}>0$, the signs of $u, v$, and $w$ must be such that

$$
\text { (i) } u, w>0 \text { and } v<0 \text { or } \quad(i i) u, w<0 \text { and } v>0 .
$$

Notice that from the first equation of each of the linear systems in (20), as well as from the third equation of both systems, we can, respectively, conclude that

$$
m_{2}=\frac{\frac{\delta}{b}}{\frac{a}{b}-\frac{u}{v}} \text { and } m_{2}=\frac{\frac{\delta}{b}}{\frac{c}{b}-\frac{w}{v}} .
$$

Then $\frac{a-c}{b}=\frac{u-w}{v}$, a condition that is independent of $\delta>0$. Two similar conditions follow by circular permutations. Therefore the systems in (20) have the same set of solutions if

$$
\frac{a-c}{b}=\frac{u-w}{v}, \quad \frac{b-a}{c}=\frac{v-u}{w}, \quad \frac{c-b}{a}=\frac{w-v}{u} .
$$

We will further prove that these conditions are simultaneously satisfied only when $a=b=c$ and $u=-v=w$, a set of solutions which corresponds to equilateral triangles and, as a result, to equal masses.

Let us first assume that the acute triangle is isosceles and not equilateral. Then, without loss of generality, we can choose $a=c \neq b$ and $u=w \neq v$. The first equation in (22) is satisfied, whereas the other two take the same form, namely $\frac{a-b}{a}=\frac{u-v}{u}$, which implies that $a v=b u$. Since $a, b>0$, it follows that $u$ and $v$ have the same sign, a conclusion which contradicts (21).

The last case to consider is that of the scalene acute triangles that are not isosceles. Without loss of generality, we assume that $0<a<b<c$. Then

$$
\frac{a-c}{b}<0, \quad \frac{b-a}{c}>0, \quad \frac{c-b}{a}>0,
$$

inequalities which, via (22), imply that

$$
\frac{u-w}{v}<0, \frac{v-u}{w}>0, \frac{w-v}{u}>0 .
$$


But relations (21) provide only four possible ways to order $u, v$, and $w$, namely: (1) $v<0<u<w$, (2) $v<0<w<u$, (3) $u<w<0<v$, and (4) $w<u<0<v$. It is easy to see that, in each case, the order and the signs of these constants contradict at least one of the inequalities given in (23), so scalene nonisosceles triangles cannot form homographic orbits either. This remark completes the proof.

\section{Geodesic scalene triangles}

In this section we restrict our study to the case $\kappa>0$ and consider a situation that was not captured in the previous section, namely the relative equilibria that rotate on great circles of a sphere generated from fixed points of the equations of motion. Such orbits do not exist for $\kappa<0$ because, as shown in [7] and [8, there are no fixed points when the bodies move in hyperbolic space.

Without loss of generality, we will analyze these orbits when the great circle is the equator, $z=0$, and the rotation takes place around the $z$-axis. This case is not captured by the system of differential equations given in equations (15) and (17) because one denominator cancels when the bodies reach the equator. When $r$ is constant, i.e. the solution is just a relative equilibrium, instead of a homographic orbit with both rotation and expansion and/or contraction, the motion on the equator can be studied separately. In this case, no cancelling denominators show up when the motion takes place on the great circle $z=0$. Nevertheless, no two bodies can be antipodal because the corresponding configuration is a singularity of system (8) (see [7] or [9] for more details), and therefore the motion doesn't exist. But acute triangles moving on the equator don't have antipodal bodies at their vertices, so singularities do not affect them.

We will first focus on fixed points lying on the equator, and show why for every acute triangle there exist masses that provide a fixed point for the equations of motion if the bodies are placed at the vertices of the triangle. Then we explain how relative equilibria can be generated from fixed points. By providing a large class of counterexamples, we also prove that not any choice of three masses generates fixed points, and consequently not any choice of three masses can form relative equilibria that move along the equator.

We can now state and prove the following result.

Theorem 4. For any acute triangle inscribed in a great circle of the sphere $S_{\kappa}^{2}$, there exist bodies of masses $m_{1}, m_{2}, m_{3}>0$ that can be placed at the vertices of the triangle such that they form a fixed point of system (8) for $n=3$ and $\kappa>0$, i.e. for the equations of motion of the curved 3-body problem in the case of positive curvature.

Proof. As mentioned earlier, we can assume that the great circle is the equator $z=0$. To form a fixed point of system (8), the initial conditions of the bodies of masses $m_{1}, m_{2}$, and $m_{3}$ must satisfy, at the initial instant $t=0$, the constraints

$$
\ddot{\mathbf{q}}_{i}(0)=\dot{\mathbf{q}}_{i}(0)=0, \quad i=1,2,3 .
$$

Let $\mathbf{q}_{i}(0)=\left(x_{i}, y_{i}, 0\right), i=1,2,3$, be the initial position of the body of mass $m_{i}, i=$ $1,2,3$, on the equator $z=0$. Using equations (8), a straightforward computation shows that the above conditions reduce to solving the linear homogeneous algebraic 
system

$$
\left\{\begin{array}{l}
q_{12} m_{2}+q_{13} m_{3}=0 \\
\bar{q}_{12} m_{2}+\bar{q}_{13} m_{3}=0 \\
q_{21} m_{1}+q_{23} m_{3}=0 \\
\bar{q}_{21} m_{1}+\bar{q}_{23} m_{3}=0 \\
q_{31} m_{1}+q_{32} m_{2}=0 \\
\bar{q}_{31} m_{1}+\bar{q}_{32} m_{2}=0
\end{array}\right.
$$

for $m_{1}, m_{2}$, and $m_{3}$, where, for $i, j=1,2,3, i \neq j$,

$$
q_{i j}=\frac{x_{j}-a_{i j} x_{i}}{\left(1-a_{i j}^{2}\right)^{3 / 2}}, \quad \bar{q}_{i j}=\frac{y_{j}-a_{i j} y_{i}}{\left(1-a_{i j}^{2}\right)^{3 / 2}}, a_{i j}=\kappa x_{i} x_{j}+\kappa y_{i} y_{j} .
$$

But the first and the second equation of the system are linearly dependent. Indeed, multiplying the first equation by $\kappa x_{1}$, the second equation by $\kappa y_{1}$, and adding the two new equations, we obtain an identity. Similarly we can prove the linear dependence of the third and fourth equations, as well as of the fifth and sixth equations. Therefore system (24) can be reduced to the linear homogeneous system

$$
\left\{\begin{array}{l}
q_{12} m_{2}+q_{13} m_{3}=0 \\
q_{21} m_{1}+q_{23} m_{3}=0 \\
q_{31} m_{1}+q_{32} m_{2}=0
\end{array}\right.
$$

in the unknowns $m_{1}, m_{2}$, and $m_{3}$.

We will further show that this system has positive solutions. To achieve this goal we will first prove that $\operatorname{det} A=0$, where $A$ is the matrix that defines system (25), namely

$$
A=\left[\begin{array}{ccc}
0 & q_{12} & q_{13} \\
q_{21} & 0 & q_{23} \\
q_{31} & q_{32} & 0
\end{array}\right]
$$

Notice first that

$$
\operatorname{det} A=q_{12} q_{23} q_{31}+q_{13} q_{21} q_{32} \text {. }
$$

But $q_{12}$ and $q_{21}$ have the same denominator, which is never zero. The same is true for the pair $q_{23}$ and $q_{32}$, as well as for the pair $q_{31}$ and $q_{13}$. Therefore to prove that $\operatorname{det} A=0$, it is enough to compute the numerator $E$ of $\operatorname{det} A$. Notice that $E$ has the form

$E=\left(x_{2}-a_{12} x_{1}\right)\left(x_{3}-a_{23} x_{2}\right)\left(x_{1}-a_{13} x_{3}\right)+\left(x_{3}-a_{13} x_{1}\right)\left(x_{1}-a_{12} x_{2}\right)\left(x_{2}-a_{23} x_{3}\right)$.

A straightforward computation leads to

$$
\begin{aligned}
E=2\left(1-a_{12}\right. & \left.a_{13} a_{23}\right) x_{1} x_{2} x_{3}+\left(a_{12} a_{23}-a_{13}\right) x_{1}^{2} x_{2}+\left(a_{13} a_{23}-a_{12}\right) x_{1}^{2} x_{3} \\
& +\left(a_{12} a_{13}-a_{23}\right) x_{1} x_{2}^{2}+\left(a_{13} a_{12}-a_{23}\right) x_{1} x_{3}^{2} \\
& +\left(a_{13} a_{23}-a_{12}\right) x_{2}^{2} x_{3}+\left(a_{12} a_{23}-a_{13}\right) x_{2} x_{3}^{2} .
\end{aligned}
$$

Without loss of generality, we can assume that $x_{1}=0$, i.e. the body of mass $m_{1}$ is fixed at the point of coordinates $\left(x_{1}, y_{1}, z_{1}\right)=\left(0, \kappa^{-1 / 2}, 0\right)$ on the sphere $S_{\kappa}^{2}$ (see Figure 10. Then $E=S x_{2} x_{3}$, where $S=-a_{13} x_{3}+a_{13} a_{23} x_{2}+a_{12} a_{23} x_{3}-a_{12} x_{2}$. Since $x_{2}, x_{3} \neq 0$, to prove that $\operatorname{det} A=0$ it is enough to show that $S=0$. Using 


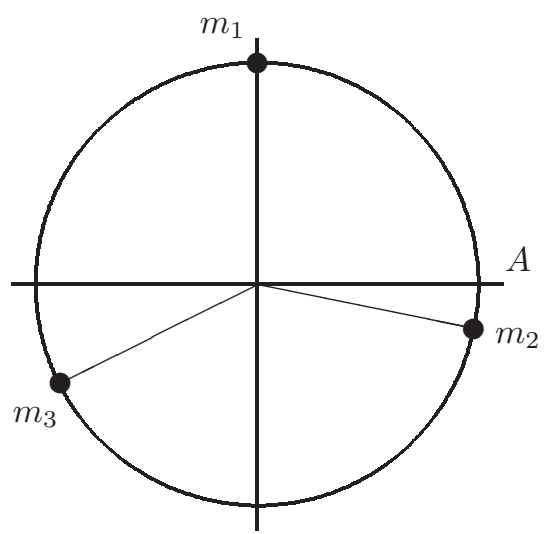

Figure 1. The fixed point solutions formed by the masses $m_{1}, m_{2}$, and $m_{3}$ on the geodesic (equator) $z=0$.

again the fact that $x_{1}=0$, and writing the constants $a_{i j}$ explicitly, we obtain

$$
\begin{aligned}
S= & -\kappa y_{1} y_{3} x_{3}+\kappa^{2} y_{1} y_{3}\left(x_{2} x_{3}+y_{2} y_{3}\right) x_{2}+\kappa^{2} y_{1} y_{2}\left(x_{2} x_{3}+y_{2} y_{3}\right) x_{3}-\kappa y_{1} y_{2} x_{2} \\
& =-\kappa x_{3} y_{1} y_{3}-\kappa x_{2} y_{1} y_{2}+\kappa x_{3} y_{1} y_{3}\left(\kappa x_{2}^{2}+\kappa y_{2}^{2}\right)+\kappa x_{2} y_{1} y_{2}\left(\kappa x_{3}^{2}+\kappa y_{3}^{2}\right) .
\end{aligned}
$$

But since $\kappa x_{2}^{2}+\kappa y_{2}^{2}=\kappa x_{3}^{2}+\kappa y_{3}^{2}=1$, it follows that $S=0$; therefore $\operatorname{det} A=0$, so system (25) has other solutions than the trivial one.

We must still prove that among these nontrivial solutions there is a class of strictly positive solutions. For this purpose, we will analyse the signs of the constants $q_{i j}, i, j=1,2,3$, and see how they determine the signs of $m_{1}, m_{2}$, and $m_{3}$. We already assumed that $x_{1}=0$. Since the triangle is acute, we can further suppose, without loss of generality, that $x_{2}>0$ and $x_{3}<0$, which means that the body of mass $m_{2}$ lies in the fourth quadrant on the equatorial circle, while $m_{3}$ is in the third quadrant (see Figure 10. There are no other possibilities because the triangle is acute. (In fact we showed in [7] and [8] that bodies lying in the same hemisphere cannot form fixed points, thus excluding right or obtuse triangles as fixed-point candidates. Right triangles are also excluded because they form singular configurations.)

Under the above assumptions, we will show that

$$
q_{12}, q_{21}, q_{32}>0 \text { and } q_{13}, q_{31}, q_{23}<0 .
$$

For this purpose, notice first that the denominators of $q_{i j}, i, j=1,2,3$, are all positive, so we have to determine only the signs of their numerators. Also observe that the angles $\alpha_{12}$ and $\alpha_{13}$ from the centre of the circle corresponding to the arcs $m_{1} m_{2}$ and $m_{1} m_{3}$, respectively (see Figure 1), both angles taken to be smaller than $\pi$, are larger than $\pi / 2$. Since $a_{12}=\cos \alpha_{12}$ and $a_{13}=\cos \alpha_{13}$, it means that $a_{12}, a_{13}<0$. Then, using the fact that $x_{1}=0, x_{2}>0, x_{3}<0$, we can conclude that $q_{12}>0$ and $q_{13}<0$. Since $a_{12}=a_{21}$ and $a_{31}=a_{13}$, it also follows that $q_{21}>0$ and $q_{31}<0$.

To prove the last two inequalities in (26), let $\beta$ be the angle from the centre of the circle corresponding to the arc $m_{2} m_{3}, \alpha$ the similar angle corresponding to the $\operatorname{arc} m_{3} A$ (see Figure 10), and $\gamma$ the similar angle corresponding to the $\operatorname{arc} m_{2} A$, all taken to be smaller than $\pi$. Then, obviously, $\alpha=\beta+\gamma$. Also notice that 


$$
\begin{aligned}
x_{3}=\cos \alpha, x_{2}= & \cos \gamma, \text { and } a_{23}=\cos \beta \text {. So } \\
& x_{3}-a_{23} x_{2}=\cos \alpha-\cos \beta \cos \gamma=-\sin \beta \sin \gamma,
\end{aligned}
$$

which is negative because $0<\beta, \gamma<\pi$. Therefore $q_{23}<0$. Finally,

$$
x_{2}-a_{23} x_{3}=\cos \gamma-\cos \beta \cos \alpha=\sin \alpha \sin \beta,
$$

which is positive because $0<\alpha, \beta<\pi$. So $q_{23}>0$. To see now that system (25) has positive solutions, it is enough to notice that the two constants $q_{i j}$ showing up in each of its three equations have opposite signs. This remark completes the proof.

A direct consequence of the above result is the possibility of generating relative equilibria from fixed points. This fact stems from the action produced by elements of the rotation group $S O(3)$ on a fixed point. From the mechanical point of view this means that we can obtain relative equilibria if we apply initial velocities, of equal speeds, tangentially to the geodesic, all oriented clockwise or all counterclockwise. This obvious remark together with Theorem 4 proves the following result.

Theorem 5. Consider the curved 3-body problem on the sphere $S_{\kappa}^{2}$, given by system (8) for $n=3$ and $\kappa>0$. Then for any acute triangle inscribed in a great circle of the sphere, there exist masses $m_{1}, m_{2}, m_{3}>0$ and initial velocities such that if the point particles are initially placed at the vertices of the triangle, the corresponding solution is a relative equilibrium that rotates on the great circle.

The converse of Theorem 4 is false, which means that not any choice of three point particles of given masses can form a fixed point on a great circle of $S_{\kappa}^{2}$. We will provide a class of counterexamples in the case of isosceles triangles. In fact, the following result identifies all masses for which there are no isosceles triangles that lead to fixed points of the equations of motion of the curved 3-body problem for $\kappa>0$.

Theorem 6. Consider the curved 3-body problem on the sphere $S_{\kappa}^{2}$, given by system (8) for $n=3, \kappa>0$, as well as the masses $m_{1}=m_{2}=: M>0$ and $m_{3}=: m>0$. Assume that the initial conditions are such that the triangle having these masses at its vertices is acute and isosceles, with the equal masses corresponding to the base. Then, for $M \geq 4 m$, no isosceles triangle can form a fixed point.

Proof. Let $\mathbf{q}_{i}(0)=\left(x_{i}(0), y_{i}(0), 0\right), i=1,2,3$, be the initial position of the masses with the symmetries of an isosceles triangle as in Figure 2, i.e. such that

$$
\begin{gathered}
x_{1}(0)=x, x_{2}(0)=-x, x_{3}(0)=0, \\
y_{1}(0)=y_{2}(0)=y, y_{3}(0)=-\kappa^{-1 / 2}, \\
z_{1}(0)=z_{2}(0)=z_{3}(0)=0,
\end{gathered}
$$

with $0<x, y<\kappa^{-1 / 2}$. The conditions that the coordinates form a fixed point are

$$
\ddot{\mathbf{q}}_{i}(0)=\dot{\mathbf{q}}_{i}(0)=0, \quad i=1,2,3 .
$$

Using these conditions in system (8), denoting $\ddot{x}:=\ddot{x}(0), \ddot{y}:=\ddot{y}(0)$ and asking that $\dot{x}(0)=\dot{y}(0)=0$, a straightforward computation leads us to the relations

$$
\ddot{x}=-\frac{M-4 \kappa m y^{2}}{4 \kappa^{1 / 2} x^{2} y}, \quad \ddot{y}=\frac{M-4 \kappa m y^{2}}{4 \kappa^{1 / 2} x y^{2}} .
$$




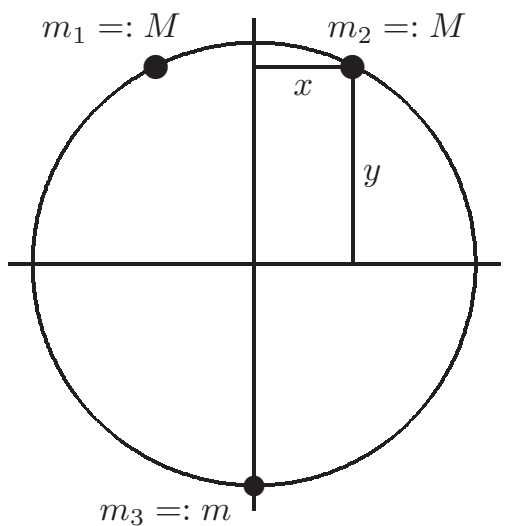

FiguRE 2. The initial positions of $m_{1}, m_{2}$, and $m_{3}$, which form an isosceles triangle on the geodesic (equator) $z=0$.

Consequently 3 bodies lying at the vertices of an isosceles triangle form a fixed point of the equations of motion if and only if

$$
M=4 \kappa m y^{2}, \text { with } 0<y<\kappa^{-1 / 2} .
$$

Then some necessary conditions that an isosceles triangle forms a fixed point are

$$
0<\frac{M}{4 \kappa m}<\frac{1}{\kappa} .
$$

Since $\kappa>0$, the first inequality is always satisfied. The second inequality reduces to $M<4 m$. The corresponding $x>0$ is then obtained from $\kappa x^{2}+\kappa y^{2}=1$ and leads to the same condition. So, for $M \geq 4 m$, there are no isosceles triangles that form fixed points on a great circle of $\mathbf{S}_{\kappa}^{2}$. This remark completes the proof.

Remark. When the triangle is equilateral, the evaluation of $\sin \varphi$, where $\varphi$ is the angle between the abscissa and the radius of the circle to $m_{2}$ (see Figure 2), shows that $y=1 /\left(2 \kappa^{-1 / 2}\right)$, so by (27) we can conclude that $M=m$, in agreement with what we know about Lagrangian solutions.

\section{ACKNOWLEDGMENT}

The research presented in this paper was supported in part by a Discovery Grant from NSERC of Canada.

\section{REFERENCES}

[1] R. Abraham and J.E. Marsden, Foundations of Mechanics (2nd ed.) Benjamin-Cummings, Reading, MA, 1978. MR515141(81e:58025)

[2] J. Bertrand, Théorème relatif au mouvement d'un point attiré vers un centre fixe, C. R. Acad. Sci. 77 (1873), 849-853.

[3] J.F. Cariñena, M.F. Rañada, and M. Santander, Central potentials on spaces of constant curvature: The Kepler problem on the two-dimensional sphere $\mathbf{S}^{2}$ and the hyperbolic plane $\mathbf{H}^{2}$, J. Math. Phys. 46 (2005), 052702. MR2143000(2006d:70019)

[4] F. Diacu, Near-collision dynamics for particle systems with quasihomogeneous potentials, $J$. Differential Equations 128 (1996), 58-77. MR1392396 (97i:70018)

[5] F. Diacu, On the singularities of the curved n-body problem, Trans. Amer. Math. Soc. 363, 4 (2011), 2249-2264. MR 2746682

[6] F. Diacu and E. Pérez-Chavela, Homographic solutions of the curved 3-body problem, J. Differential Equations 250 (2011), 340-366. MR2737846 
[7] F. Diacu, E. Pérez-Chavela, and M. Santoprete, The $n$-body problem in spaces of constant curvature, arXiv:0807.1747, $54 \mathrm{pp}$.

[8] F. Diacu, E. Pérez-Chavela, and M. Santoprete, The $n$-body problem in spaces of constant curvature. Part I: Relative equilibria, J. Nonlinear Sci. (to appear).

[9] F. Diacu, E. Pérez-Chavela, and M. Santoprete, The $n$-body problem in spaces of constant curvature. Part II: Singularities, J. Nonlinear Sci. (to appear).

[10] F. Diacu, E. Pérez-Chavela, and M. Santoprete, Saari's conjecture for the collinear $n$-body problem, Trans. Amer. Math. Soc. 357, 10 (2005), 4215-4223. MR2159707(2007k:70014)

[11] F. Diacu, T. Fujiwara, E. Pérez-Chavela, and M. Santoprete, Saari's homographic conjecture of the 3-body problem, Trans. Amer. Math. Soc. 360, 12 (2008), 6447-6473. MR 2434294 (2010f:70010)

[12] A. Einstein, L. Infeld, and B. Hoffmann, The gravitational equations and the problem of motion, Ann. of Math. (2) 39, 1 (1938), 65-100. MR.1503389

[13] B. Elmabsout, Sur l'existence de certain configurations d'équilibre relatif dans le problème des $N$ corps, Celest. Mech. Dyn. Astr. 41 (1988), 131-151. MR 954880 (89j:70014)

[14] V. A. Fock, Sur le mouvement des masses finie d'après la théorie de gravitation einsteinienne, J. Phys. Acad. Sci. USSR 1 (1939), 81-116. MR0001124 (1:183a)

[15] G. T. Gilbert, Positive definite matrices and Sylvester's criterion, Amer. Math. Monthly 98, 1 (1991), 44-46. MR1083614 (92a:15009)

[16] L. Infeld, On a new treatment of some eigenvalue problems, Phys. Rev. 59 (1941), 737-747. MR.0004347 (2:364a)

[17] L. Infeld and A. Schild, A note on the Kepler problem in a space of constant negative curvature, Phys. Rev. 67 (1945), 121-122. MR0011962 (6:242b)

[18] W. Killing, Die Rechnung in den nichteuklidischen Raumformen, J. Reine Angew. Math. 89 (1880), 265-287.

[19] W. Killing, Die Mechanik in den nichteuklidischen Raumformen, J. Reine Angew. Math. 98 (1885), 1-48.

[20] W. Killing, Die Nicht-Eukildischen Raumformen in Analytischer Behandlung, Teubner, Leipzig, 1885.

[21] T. Levi-Civita, The relativistic problem of several bodies, Amer. J. Math. 59, 1 (1937), 9-22. MR 1507213

[22] T. Levi-Civita, Le problème des n corps en relativité générale, Gauthier-Villars, Paris, 1950; or the English translation: The $n$-body problem in general relativity, D. Reidel, Dordrecht, 1964. MR0044932 (13:499d)

[23] H. Liebmann, Die Kegelschnitte und die Planetenbewegung im nichteuklidischen Raum, Berichte Königl. Sächsischen Gesell. Wiss., Math. Phys. Klasse 54 (1902), 393-423.

[24] H. Liebmann, Über die Zentralbewegung in der nichteuklidische Geometrie, Berichte Königl. Sächsischen Gesell. Wiss., Math. Phys. Klasse 55 (1903), 146-153.

[25] H. Liebmann, Nichteuklidische Geometrie, G. J. Göschen, Leipzig, 1905; 2nd ed. 1912; 3rd ed. Walter de Gruyter, Berlin, Leipzig, 1923.

[26] R. Lipschitz, Extension of the planet-problem to a space of $n$ dimensions and constant integral curvature, Quart. J. Pure Appl. Math. 12 (1873), 349-370.

[27] J.E. Marsden and T.S. Ratiu, Introduction to Mechanics and Symmetry. (2nd ed.) Springer, 1999. MR 1723696(2000i:70002)

[28] A. I. Miller, The myth of Gauss's experiment on the Euclidean nature of physical space, Isis 63, 3 (1972), 345-348. MR0532182 (58:27030)

[29] L. Perko and E. Walter, Regular polygon solutions of the $N$-body problem, Proc. Amer. Math. Soc. 94 (1985), 301-309. MR784183 (86e:70004)

[30] D. Saari, Collisions, Rings, and Other Newtonian N-Body Problems, American Mathematical Society, Regional Conference Series in Mathematics, No. 104, Providence, RI, 2005. MR2139425 (2006d:70030)

[31] E. Schering, Die Schwerkraft im Gaussischen Räume, Nachr. Königl. Gesell. Wiss. Göttingen 13 July, 15 (1870), 311-321.

[32] E. Schering, Die Schwerkraft in mehrfach ausgedehnten Gaussischen und Riemmanschen Räumen, Nachr. Königl. Gesell. Wiss. Göttingen 26 Febr., 6 (1873), 149-159.

[33] E. Schrödinger, A method for determining quantum-mechanical eigenvalues and eigenfunctions, Proc. Royal Irish Acad., Sect. A 46 (1940), 9-16. MR0001666 (1:277d) 
[34] A. Wintner, The Analytical Foundations of Celestial Mechanics, Princeton University Press, Princeton, N.J., 1941. MR0005824(3:215b)

Pacific Institute for the Mathematical Sciences - and - Department of Mathematics and Statistics, University of Victoria, P.O. Box 3060 STN CSC, Victoria, British Columbia, Canada V8W 3R4

E-mail address: diacu@math.uvic.ca 